ÉGYPTE monde arabe

\section{Égypte/Monde arabe}

2 | 2005

Les architectures constitutionnelles des régimes

politiques arabes

\title{
Les architectures constitutionnelles : introduction
}

\section{Nathalie Bernard-Maugiron et Jean-Noël Ferrié}

\section{(2) OpenEdition}

Journals

Édition électronique

URL : https://journals.openedition.org/ema/1049

DOI : 10.4000/ema.1049

ISSN : 2090-7273

Éditeur

CEDEJ - Centre d'études et de documentation économiques juridiques et sociales

Édition imprimée

Date de publication : 31 décembre 2005

Pagination : 7-16

ISSN : 1110-5097

Référence électronique

Nathalie Bernard-Maugiron et Jean-Noël Ferrié, « Les architectures constitutionnelles : introduction », Égypte/Monde arabe [En ligne], 2 | 2005, mis en ligne le 08 juillet 2008, consulté le 07 juillet 2022. URL http://journals.openedition.org/ema/1049; DOI : https://doi.org/10.4000/ema.1049

Ce document a été généré automatiquement le 7 juillet 2022

Tous droits réservés 


\title{
Les architectures constitutionnelles : introduction
}

\author{
Nathalie Bernard-Maugiron et Jean-Noël Ferrié
}

\section{NOTE DE L'AUTEUR}

Le colloque qui a précédé cette publication a bénéficié d'une subvention du CNRS, accordée par la section 40 du comité national, en 2004. Il n'aurait pu être organisé sans l'aide d'Assia Boutaleb et de Benjamin Rey.

\section{Le mouvement constitutionnel dans le monde arabe}

1 Après s'être longtemps désintéressée de l'étude des institutions, au profit notamment de l'étude de l'islam politique, la recherche sur le monde arabo-musulman ${ }^{1}$ commence à découvrir les vertus de l'étude des textes fondamentaux. Des recueils de constitutions arabes voient le jour (Canal-Forgues, 2000), des colloques sur les constitutions des pays arabes sont organisés (CEDROMA, 1999), les conditions d'apparition de constitutions dans le monde arabe et leur lien ou non avec le constitutionnalisme sont analysées (Brown 2002), les parlements arabes font l'objet d'études comparées (Baaklini et al., 1999). Les cours constitutionnelles de pays arabes (Bendourou 1997, Ben Achour 1990 et 1995, Mallat 1993) et notamment la plus active d'entre elles, la Haute Cour constitutionnelle égyptienne, font l'objet d'ouvrages spécifiques (Bernard-Maugiron et Dupret 1999a ; Boyle et Sherif 1996).

2 À l'heure actuelle, tous les pays du monde arabe se sont dotés de textes écrits organisant le pouvoir, qu'ils les aient intitulés Constitution (dustûr) (Algérie, Bahreïn, Égypte, Émirats arabes unis, Irak, Jordanie, Koweït, Liban, Maroc, Mauritanie, Soudan, Syrie, Tunisie, Yémen) ou "Statut fondamental (nizâm asâsi)» (Arabie saoudite, Oman, Qatar). L'Arabie saoudite a adopté en 1992 un statut fondamental (nizâm asâsi lîl-l-hukm) organisant la forme du gouvernement, ainsi que deux autres ordonnances, 
réorganisant le shura council et portant sur l'organisation des régions. Oman a fini par suivre le mouvement en 1996. La fin du xxe siècle a également été marquée par des réformes constitutionnelles (Algérie 1989 et 1996, Liban 1990, Maroc 1992 et 1996, Tunisie 1996). En 1996, la Constitution provisoire des Émirats est devenue permanente. En 2002, deux pays ont déjà procédé à des réformes constitutionnelles : la Constitution tunisienne a ainsi été révisée en mai 2002 pour permettre le renouvellement du mandat présidentiel, et la Constitution du Bahreïn a été modifiée en février 2002 pour changer le statut de l'Émirat en monarchie, rétablir le parlementarisme, mettre en place des conseils municipaux élus et donner aux femmes le droit d'éligibilité. La Constitution du Qatar est en cours de modification, un comité ayant été nommé pour élaborer un nouveau texte. La Libye est le seul État à n'avoir pas matériellement de constitution ou de loi fondamentale, mais elle a toutefois promulgué des textes définissant l'organisation des pouvoirs selon des règles préétablies. La déclaration de remise du pouvoir au peuple (1977) lui tient en quelque sorte lieu de constitution.

Ces constitutions ont presque toujours été instituées par les gouvernants eux-mêmes. Elles ont été le plus souvent élaborées par un comité gouvernemental, souvent étroitement lié au souverain en place (Jordanie 1952, Émirats 1971, Qatar 1972, Maroc 1992 et 1996, Arabie saoudite 1992, Oman 1996). Il est très rare que la constitution ait été élaborée par des représentants élus (Tunisie 1959, Koweït 1962, Égypte 1971, Bahreïn 1973). Enfin, les textes sont généralement octroyés par le prince, consécration de son hégémonie politique (Jordanie 1952, Koweït 1962), bien qu'ils soient parfois soumis à référendum (Syrie 1973, Mauritanie 1991, Maroc 1996, Algérie 1996, révision constitutionnelle tunisienne 2002).

\section{Les constitutions ou comment les contourner}

\section{Mise en place d'institutions démocratiques}

4 Les pays de la région présentent de grandes disparités de formes politiques et leurs constitutions présentent une remarquable diversité dans leur contenu et dans leur forme, distribuant les compétences et organisant le pouvoir selon des modèles variés. L'éventail des régimes politiques va ainsi des monarchies (Arabie saoudite, Bahreïn, Jordanie, Maroc) aux républiques (Algérie, Égypte, Irak), en passant par les émirats (Émirats arabes unis, Koweït, Qatar) ou même un sultanat (Oman). On retrouve toutefois dans tous ces textes les structures modernes du pouvoir et un grand nombre de caractéristiques des modes d'organisation politiques connus en Occident.

Qu'ils empruntent davantage au système parlementaire ou présidentiel, tous se caractérisent par une forte concentration du pouvoir aux mains de l'exécutif. Ils consacrent en général la primauté du chef de l'État, qu'il soit roi, émir, sultan ou président. Toujours irresponsable politiquement, il nomme et révoque les ministres et est parfois doté de l'exclusivité du pouvoir législatif (Oman, Arabie saoudite, Qatar, Émirats arabes unis) voire même d'une certaine autorité vis-à-vis du pouvoir judiciaire (Arabie saoudite). Le recours à la forme constitutionnelle n'a bien souvent pas entraîné de changement fondamental dans l'organisation du pouvoir et revêt donc une signification immédiate limitée. Il serait abusif de considérer ces textes fondamentaux comme représentant un frein à l'absolutisme, limitant l'exercice du pouvoir à travers une régulation du fonctionnement des pouvoirs publics. Au contraire, ils ont souvent 
consacré la pérennisation du pouvoir, en codifiant les principes d'exercice du pouvoir pratiqués jusqu'alors par les régimes en place.

6 Bien qu'issus du pouvoir en place et caractérisés par la prévalence de l'autoritarisme, ces textes, inspirés du régime représentatif, mettent souvent en place des institutions démocratiques. Plusieurs d'entre eux, particulièrement les régimes républicains, instituent une assemblée représentative, élue par le peuple (Algérie, Bahreïn, Égypte, Koweït, Maroc, Syrie, Tunisie), dotée de pouvoirs législatifs et de pouvoirs de contrôle. Les ministres y sont responsables devant le législatif, individuellement (Bahreïn, Koweït) ou collectivement (Algérie, Égypte, Jordanie, Liban, Maroc, Syrie, Tunisie), et les lois ne peuvent être adoptées sans le consentement de l'assemblée (Algérie, Bahreïn, Égypte, Jordanie, Koweït, Liban, Tunisie). D'autres pays, particulièrement les monarchies du Golfe (Émirats arabes unis, Oman, Qatar) et l'Arabie saoudite, continuent toutefois de refuser tout organe parlementaire représentatif, se contentant d'une assemblée consultative nommée par le souverain.

\section{Les constitutions, contraintes organisationnelles}

7 Ces textes fondamentaux des pays arabes, de même que les régimes politiques qu'ils mettent en place, sont souvent ignorés ou méconnus. On a souvent tendance à n'y voir que des textes nominaux, des constitutions de papier n'ayant aucun effet sur l'exercice du pouvoir, des instruments de légitimation du pouvoir à usage interne et externe destinés à donner un vernis démocratique national ou international à leur régime. À la différence des constitutions normatives, les constitutions des pays arabes n'auraient aucune influence réelle sur l'exercice du pouvoir, se contentant de poser des principes généraux très souvent violés dans la pratique.

Si les constitutions n'étaient effectivement que des façades, alors les régimes en place les violeraient allègrement et impunément. Or, en pratique, il est rare qu'une constitution soit violée ouvertement. En général, les organes du pouvoir respectent les règles posées par le texte constitutionnel, qui leur octroie d'ailleurs un grand nombre de pouvoirs. Pourquoi le régime violerait-il une constitution qui expose souvent ouvertement, naïvement (?), la réalité de l'exercice du pouvoir? Même lorsque la constitution limite ses compétences et qu'il veut néanmoins intervenir dans la sphère de compétence des autres pouvoirs, le souverain respecte en général les formes constitutionnelles. Une façon d'échapper aux limites fixées par la constitution consistera à moduler l'interprétation de ses dispositions de façon restrictive ou extensive, selon les cas. La mise en place d'architectures constitutionnelles démocratiques dans les pays arabes impose aux gouvernants autoritaires d'agir dans les limites du cadre institutionnel qu'ils ont tracé et de se conformer à la référence démocratique dans laquelle ils se sont situés. Ils ne les tiennent pas pour une simple façade puisqu'ils s'efforcent au moins de respecter les formes. Les règles qui déterminent le fonctionnement des institutions sont contraignantes en ce sens qu'elles influent fortement sur les dynamiques dans lesquelles les acteurs sont engagés. Les architectures constitutionnelles, en tant que mécanismes, ont donc un caractère contraignant ; les interactions vont être contraintes par les règles institutionnelles. 


\section{Détournement des contraintes constitutionnelles}

Si les règles institutionnelles posent des contraintes, les acteurs du champ politique ont cependant appris à les surmonter. Ces institutions démocratiques, en effet, ont été mises en place par un acteur dominant qui a rarement intérêt à les voir pleinement fonctionner et qui va les promouvoir, tout en falsifiant leur fonctionnement pour pouvoir perdurer. Les gouvernants conservent ainsi la maîtrise quasi absolue de l'architecture constitutionnelle et aucun opposant n'est en mesure de leur imposer cette démocratisation qu'ils ont octroyée.

Plutôt que de violer ouvertement la constitution, le pouvoir en place préfèrera la suspendre en tout ou en partie. Le chef de l'État recourra parfois aux pouvoirs exceptionnels que lui octroie la constitution, allant jusqu'à déclarer ou prolonger indéfiniment un état d'urgence sous couvert de protection de la sécurité et de l'ordre public. C'est le cas en Égypte, ce fut le cas en Syrie, en Iraq, en Jordanie et au Soudan. Lorsqu'une assemblée parlementaire cherche trop ouvertement à exercer la totalité des pouvoirs qui lui ont été conférés par la loi fondamentale, il arrive aussi que le chef de l'État suspende les dispositions constitutionnelles y relatives. Cette méthode avait déjà été utilisée par le Roi Fu'âd en Égypte dans les années vingt. Les Émirs du Bahrein et du Koweït y ont également eu recours. En 1930, le Roi Fu'ad avait même fini par abroger la Constitution de 1923 et par la remplacer par une constitution très proche mais qui renforçait ses pouvoirs. Quant Bechir al-Assad a pris le pouvoir en Syrie à la mort de son père, la Constitution fut amendée afin de diminuer l'âge minimum requis pour exercer cette fonction.

Un certain nombre d'États arabes ont aussi choisi d'amender leur constitution afin d'y introduire une deuxième chambre (Égypte 1980, Bahreïn 1992, Algérie 1996, Maroc 1996, Tunisie 2002). L'introduction d'un parlement bicaméral a souvent pour effet de diviser le pouvoir législatif, en permettant à une chambre haute, nommée souvent par scrutin indirect, de faire contrepoids à la chambre basse.

Une des dispositions qui gêne le plus un président en place est celle qui lui impose de quitter le pouvoir. Il arrive, en effet, que la constitution limite le nombre des mandats présidentiels ou fixe un âge limite pour pouvoir se présenter à la tête de l'État. Là encore, plutôt que de violer ouvertement la constitution, le chef de l'État va en respecter la lettre (sinon l'esprit) : afin de rester au pouvoir, il va l'amender. C'est ainsi que depuis 1980 la Constitution égyptienne ne limite plus le nombre de mandats présidentiels (art. 74). La Tunisie est également symptomatique de ce type d'expédients : en 1975, Bourguiba amendait la Constitution pour se nommer président à vie. Ben Ali, arrivé au pouvoir en 1987 dans le respect formel des formes constitutionnelles (art. 57), amende le même article en 1988 pour supprimer la présidence à vie, instaurer une limite d'âge pour se présenter (70 ans) et limiter à 3 le nombre de mandats (art. 39 et 40). En 2002, par un curieux retour de balancier de l'histoire, le président Ben Ali amenda à nouveau cet article pour supprimer la limite du nombre de mandats et repousser la limite d'âge de 70 à 75 ans.

Les constitutions mettant très souvent en place un régime représentatif, l'acteur dominant va bien organiser des élections, mais tout en évitant de les perdre sans totalement les truquer, faute de quoi le bénéfice même qu'il retire de leur organisation disparaîtrait. L'obligation constitutionnelle d'organiser des élections sera respectée car ceux-la même qui les dénaturent pensent tout de même ne pas pouvoir s'en passer pour 
asseoir leur pouvoir. Mais les élections seront falsifiées et les préférences de la population détournées, alors que les formes constitutionnelles seront respectées. Il y aura falsification des élections mais élections quand même. L'exécutif a aussi eu recours à d'autres formes d'intervention pour parer au danger que pourrait représenter une assemblée législative trop indépendante. En Algérie, le processus électoral fut ainsi stoppé avant le second tour des élections devant les risques de victoire du FIS.

\section{Les facteurs d'un changement politique}

Si l'architecture constitutionnelle favorise dans un premier temps la pérennité de l'autoritarisme, dans un deuxième temps elle peut toutefois favoriser la démocratisation. Ces architectures constitutionnelles représentatives entraînent, en effet, un risque permanent de dérapage, même si elles sont utilisées pour manipuler et contenir l'expression des préférences des gouvernés. Les gouvernements, en effet, ne sont pas assurés de contrôler les mécanismes mis en place, malgré toutes les précautions qu'ils prennent pour y parvenir. Il existe un risque inhérent à la mise en place d'institutions représentatives pluralistes dans un régime qui n'entend pas se départir vraiment de son caractère autoritaire : leur évolution est toujours incertaine parce qu'elle dépend d'une multitude d'acteurs poursuivant des intérêts particuliers.

\section{Émancipation des institutions}

Les institutions créent les conditions de leur fonctionnement. Elles finissent par créer les comportements nécessaires à leur fonctionnement ou, plus exactement, les nécessités organisationnelles liées aux contextes institutionnels font peser des contraintes sur les acteurs, les amenant à orienter leurs comportements en fonction des caractéristiques manifestes des institutions à l'intérieur desquelles ils interagissent. Les institutions et les normes démocratiques mises en place par les dirigeants autoritaires ont leur propre efficacité, en ce sens qu'elles transportent avec elles un ensemble de contraintes qui redéfinissent les situations locales indépendamment de l'engagement des acteurs.

Derrière les institutions se trouvent des acteurs politiques, des groupes qui vont chercher à accroître leur pouvoir. La pratique peut alors être différente de l'intention originelle des constituants. On l'a vu avec le Conseil constitutionnel français ou la Cour constitutionnelle égyptienne. Certaines assemblées parlementaires, de même, ont joué un rôle non négligeable et tenté d'utiliser toutes leurs prérogatives, voire même d'étendre le champ de leurs compétences, afin de peser effectivement sur les politiques publiques. Elles doivent toutefois rester prudentes car l'expérience a montré que lorsqu'une assemblée entend exercer trop rapidement les pouvoirs qui lui ont été conférés, son existence même est en danger.

17 Les assemblées législatives qui avaient été mises en place au Bahreïn (Constitution de 1973) et au Koweït (Constitution de 1962) ont ainsi fonctionné un certain temps, avant qu'une crise constitutionnelle n'éclate et qu'elles ne soient suspendues ou dissoutes par le chef de l'État (1975 au Bahreïn ; 1976 puis 1986 au Koweït). Ces assemblées avaient, en effet, eu le tort de vouloir utiliser les pouvoirs qui leur avaient été conférés par la constitution, notamment la possibilité de retirer leur confiance aux membres du gouvernement. Les dispositions constitutionnelles appelant à des élections 
automatiques après la dissolution de l'Assemblée furent suspendues dans les deux cas. De nouvelles élections furent finalement organisées au Koweït en 1981, mais l'Assemblée fut à nouveau dissoute en 1986. Une nouvelle Assemblée fut élue en 1996 avant d'être à son tour dissoute en 1999, dans les formes constitutionnelles. Au Bahreïn, l'amendement constitutionnel de février 2002 a rétabli le parlementarisme et des élections à la chambre basse du Parlement ont eu lieu en octobre 2002.

Le même sort fut réservé à l'Assemblée nationale jordanienne, ajournée de 1973 à 1989, en raison d'obstacles tenant à l'organisation des élections. Cette pratique était apparue dès les débuts du parlementarisme dans le monde arabe. Ainsi, le Roi Fu'ad, excédé de voir le Wafd remporter systématiquement les élections à la chambre basse du Parlement avait décidé, déjà, de ne plus réunir le Parlement en 1926 pour une durée de 3 ans, et avait adopté un décret suspendant les articles de la constitution y relatifs.

19 Au Maroc, après l'adoption de la Constitution de 1962, l'état d'urgence fut proclamé en 1965 afin de suspendre l'Assemblée, jusqu'en 1970. Là encore, les formes constitutionnelles (art. 35) étaient respectées.

Les dirigeants s'efforcent en général de recouvrir leurs décisions d'un vernis de légalité. La décision de suspendre l'Assemblée est illégale mais est prise dans les formes juridiques. Ils s'efforcent de rendre le droit conforme aux faits, quitte à distordre quelque peu le cadre juridique. À chaque fois, cependant, le souverain a fini par rétablir le processus constitutionnel et, par ce, se résoudre à convoquer à nouveau les électeurs pour l'élection d'une nouvelle Assemblée nationale.

21 Les régimes en place vont souvent tenter de limiter la portée des dispositions constitutionnelles en adoptant des lois d'application restrictives. La Constitution du Qatar, par exemple, prévoit l'élection d'une chambre mais les élections n'ont jamais pu être organisées parce que la loi électorale n'a jamais été adoptée. Le mode de scrutin électoral peut être choisi de façon à pouvoir garder le contrôle des résultats, des conditions peuvent être imposées au droit de vote et de candidature. Par ailleurs, le règlement intérieur des assemblées peut limiter les moyens effectifs de contrôle de l'exécutif par les représentants du peuple, limitant la fréquence des questions et interpellations adressées aux ministres, allongeant le délai imparti aux ministres pour répondre, exigeant que l'interpellation soit introduite par un nombre minimum de députés, interdisant qu'elle débouche sur le vote d'une motion de censure. Il peut aussi donner la maîtrise de l'ordre du jour au parti majoritaire ou autoriser le président de l'Assemblée à limiter arbitrairement les prises de parole des députés.

Outre rester au pouvoir et contrôler les institutions représentatives, une troisième préoccupation des dirigeants en place est d'éviter l'apparition d'un parti d'opposition dominant. La plupart des régimes politiques arabes reconnaissent au moins formellement le multipartisme. Certes, les partis sont totalement absents de la vie politique aux Émirats, Oman, Bahreïn, Koweït et la Constitution syrienne attribue toujours un rôle dirigeant au parti Baath (art. 8). Mais la Constitution égyptienne de 1971 (art. 5), après avoir elle aussi mis en place un régime de parti unique, a été amendée en 1980 pour instituer le pluripartisme. De même, la Constitution algérienne fut amendée en 1989 (art. 42) pour reconnaître le droit de créer des associations à caractère politique, renommées partis politiques en 1996, et le Maroc est connu pour son multipartisme. Comment les partis politiques s'insèrent-ils dans la contrainte organisationnelle mise en place par la constitution? Pour contourner le multipartisme constitutionnel, les pouvoirs en place ont très souvent mis en place des conditions 
draconiennes de création d'un parti politique. D'autres interdissent certains partis, notamment ceux fondés sur une base religieuse (ex. art. 42.3 de la Constitution algérienne telle qu'amendée en 1996, loi égyptienne de 1977 sur les partis politiques) ou bien le Parti communiste (Jordanie 1953). L'Égypte, quant à elle, permet à un organe administratif de dissoudre ou de geler les activités des partis politiques pour des motifs tenant parfois à de simples dissensions internes au parti.

Enfin, le contrôle de constitutionnalité est en plein développement dans le monde arabe depuis les années 70. Le Koweït (art. 173 Constitution de 1962, mis en œuvre par loi de 1973), l'Égypte (art. 175 de la Constitution, mis en œuvre par loi de 1979), la Tunisie (décret de 1987 puis lois constitutionnelles de 1995 et 1998), l'Algérie (1989), le Maroc (Constitution de 1992, mise en œuvre par loi de 1994), la Mauritanie (1991), la Syrie (Constitution de 1973), les Émirats arabes unis (1971), le Liban (amendement constitutionnel de 1990, mis en œuvre par loi de 1993) et le Yémen (Constitution de 1991) ont ainsi mis en place des modes de justice constitutionnelle. Mais les dispositions constitutionnelles ne font bien souvent que poser le principe de la mise en place d'une cour constitutionnelle, laissant à une loi à venir le soin d'en fixer les détails. Or l'indépendance et le pouvoir effectif de la cour vont dépendre en grande partie de cette loi d'application. Les conditions de nomination, durée du mandat, immunités particulières des juges, le mode de saisine de la cour, les textes qui pourront faire l'objet d'un recours, l'effet de ses décisions, tous ces aspects fondamentaux seront tributaires du législateur. Ces juridictions se voient-elles interdire de connaître des "actes de souveraineté", permettant ainsi à des textes particulièrement sensibles d'échapper à tout contrôle juridictionnel? Quelle est la portée de la mise en place de ces juridictions constitutionnelles? Constituent-elles des contraintes à prendre en considération? Peut-on envisager qu'elles connaissent un développement semblable a celui qu'a connu la Haute Cour constitutionnelle égyptienne?

\section{Risques de " dérapage démocratique »}

Après avoir fait appel aux notions de constitutionnalisme, démocratie et état de droit, les régimes vont devoir agir dans les limites du cadre qu'ils ont fixé s'ils veulent préserver un minimum de crédibilité. En adoptant la rhétorique de la démocratie et des droits de l'homme, ils doivent accepter que leurs pratiques soient mesurées à l'aune de ces critères. Ils vont devoir se soumettre à ces contraintes organisationnelles et limiter leurs ressources de justification aux dévoiements que cette référence peut accepter sans être contredite de manière flagrante.

La libéralisation des régimes autoritaires n'est pas conçue par ses instigateurs comme devant conduire à la démocratie (puisqu'ils ne veulent surtout pas perdre le pouvoir). Mais en adoptant les structures modernes de la légalité constitutionnelle, les régimes politiques arabes ne se seraient-ils pas engagés malgré eux dans un processus de mutation des formes d'exercice du pouvoir? Le contrôle du jeu ne risque-t-il pas, à terme, d'échapper au pouvoir en place ? L'extension de la libéralisation peut entraîner des "dérapages démocratiques", c'est-à-dire créer des conduites démocratiques là où elle ne visait qu'à élargir, moyennant des contreparties plus ou moins substantielles, l'assiette des gouvernants. Les normes démocratiques produisent ainsi des effets du fait même de leur adoption, indépendamment de ce que pensent leurs importateurs. Le changement institutionnel va entraîner un changement dans l'attitude des acteurs et 
susciter de nouveaux engagements. L'adoption d'institutions démocratiques crée, à plus ou moins court terme, des comportements favorables à la démocratie.

Par ailleurs, l'extension des domaines d'intervention de l'État entraîne l'extension de ses activités et l'association d'un nombre de plus en plus grand d'institutions à l'exercice du pouvoir. Certes, le pouvoir est toujours centralisé, mais il doit être partagé entre un nombre croissant d'institutions, ce qui entraîne également une plus grande complexité de l'organisation étatique. Le pouvoir contrôle de plus en plus de secteurs d'activités, mais en contrepartie associe de plus en plus de personnes à l'exercice du pouvoir.

Certes, jusqu'à présent, un petit nombre seulement des institutions constitutionnelles ont pu effectivement jouer un rôle de régulation. Mais à la faveur d'un changement de rapport de forces, une nouvelle interprétation d'un même texte fondamental n'est-elle pas envisageable? Voire, à terme, l'amendement de la loi fondamentale ou son remplacement par une nouvelle constitution, plus respectueuse de l'équilibre des pouvoirs? Dans les pays où un minimum d'équilibre des pouvoirs a été instauré par le texte fondamental, ces dispositions, mêmes nominales, ne sont-elles pas susceptibles d'accéder à la normativité ? Les citoyens eux-mêmes ne vont-ils pas invoquer l'existence des ces règles et principes écrits pour revendiquer un assouplissement du caractère absolu de l'État? C'est en ce sens que les architectures constitutionnelles (constitutional design) participent efficacement à la structuration de la vie politique. Le changement institutionnel va entraîner un changement des comportements civiques et modeler des comportements conformes à la préservation des institutions démocratiques. Les gouvernants devront-ils peut-être délibéraliser à temps, s'ils veulent éviter tout « dérapage démocratique »?

En consacrant ce numéro d'Égypte/Monde arabe aux architectures constitutionnelles des régimes politiques arabes et à leur influence organisationnelle, on s'interrogera simultanément sur les mécanismes de maintien de l'autoritarisme et les dispositifs de démocratisation. Les constitutions désignent les acteurs et les dotent de ressources, ces ressources contraignent les actions des autres acteurs, qui vont adopter différents types de stratégies pour contourner ces contraintes institutionnelles.

Une série d'études empiriques permettra d'analyser le jeu des acteurs politiques en contexte, à travers notamment le jeu des partis ou à l'occasion d'une élection ou d'une crise politique. Ces études d'interactions entre les institutions étatiques et la manière dont sont désignés leurs titulaires (système électoral, système partisan) devrait permettre d'étudier comment agissent ces mécanismes institutionnels du maintien de l'autoritarisme et comment ils se défont. Comment ils interagissent avec un processus de démocratisation, amorcé à travers certaines institutions qui ont réussi à utiliser au maximum les pouvoirs qui leur avaient été concédés par la loi fondamentale.

Cette étude de la structuration des phénomènes politiques par les formes juridiques permettra de déplacer l'analyse, qui a longtemps eu tendance à interroger les blocages du processus de démocratisation au niveau des structures sociétales. L'étude des constitutions et de leur mise en œuvre devrait, en effet, permettre également de mieux percevoir les instruments de l'autoritarisme dans certains des pays du monde arabomusulman et de questionner l'invocation de facteurs culturels ou religieux comme modes d'explication de la lenteur du processus de démocratisation. La persistance de l'autoritarisme n'est peut-être pas due, en effet, à un effet de «mentalité » mais au jeu des contraintes contextuelles découlant du choix de l'architecture constitutionnelle. 


\section{BIBLIOGRAPHIE}

BaAklini A., G. Denoeux, R. Springborg, 1999, Legislative Politics in the Arab World: The Resurgence of Democratic Institutions, Boulder et Londres, Lynne Rienne Publishers.

Ben Achour R. et A. LachaAl, 1990, « Maghreb. Le contrôle de la constitutionnalité des lois. (état de la question) », AIJC, VII.

Ben Achour R., 1995, « Extension et développement des organes de contrôle de la constitutionnalité au Maghreb », AIJC, Economica-PUAM, XI.

Bendourou O., 1997, « Conseils constitutionnels et État de droit au Maghreb », dans Mahiou A. (dir.), L'État de droit dans le monde arabe, Paris, CNRS Éditions.

Bernard-Maugiron N. et B. Dupret, 1999a, « Le Prince et son juge », Égypte/Monde arabe, nº 2.

BoyLe K. et A.O. SHERIF (dirs), 1996, Human Rights and Democracy: The Role of the Supreme Constitutional Court of Egypt, La Haye, Kluwer Law International, CIMEL Book Series $n^{\circ} 3$.

BRown N., 2002, Constitutions in a non-constitutional world. Arab Basic Laws and the Prospects for Accountable Government, Albany, State University of New York Press.

CAnal-Forgues E. (dir.), 2000, Recueil des constitutions des pays arabes, Bruxelles, Bruylant.

CEDROMA, 1999, Les Constitutions des pays arabes, Colloque de Beyrouth 1998, Bruxelles, Bruylant.

Mallat C., 1993, « Constitutional Law in the Middle East : the Emergence of judicial power », SOAS Law Department, working paper $\mathrm{n}^{\circ} 3$, University of London.

\section{NOTES}

1. Par États du monde arabo-musulman, on entendra les États membres de la Ligue arabe, à l'exclusion toutefois de la Somalie et de Djibouti, pays non arabophones, soit Algérie, Arabie saoudite, Bahrein, Égypte, Émirats arabes unis, Irak, Jordanie, Koweït, Liban, Libye, Maroc, Mauritanie, Oman, Qatar, Soudan, Syrie, Tunisie et Yémen.

\section{INDEX}

Mots-clés : droit constitutionnel, histoire constitutionnelle, Constitution

\section{AUTEURS}

NATHALIE BERNARD-MAUGIRON

Cedej 
JEAN-NOËL FERRIÉ

Cedej 